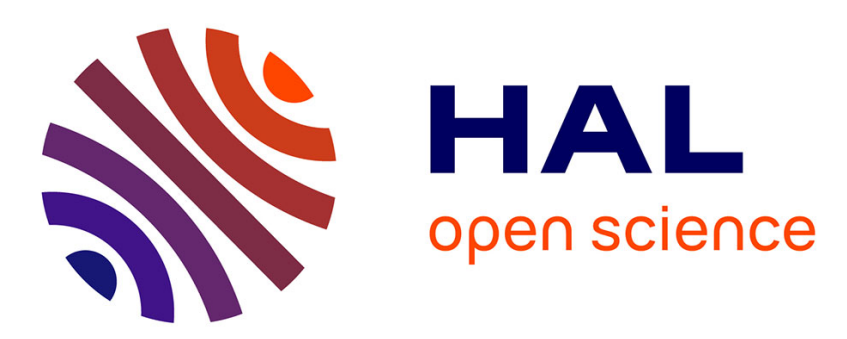

\title{
Medical knowledge modeling in a symbolic-connectionist perspective
}

\author{
Vincent Rialle, M. Ohayon, Bernard Amy, Pierre Bessiere
}

\section{To cite this version:}

Vincent Rialle, M. Ohayon, Bernard Amy, Pierre Bessiere. Medical knowledge modeling in a symbolicconnectionist perspective. sdf, 1991, France. pp.Vol. 13 - N³. hal-00089358

\section{HAL Id: hal-00089358 \\ https://hal.science/hal-00089358}

Submitted on 17 Sep 2006

HAL is a multi-disciplinary open access archive for the deposit and dissemination of scientific research documents, whether they are published or not. The documents may come from teaching and research institutions in France or abroad, or from public or private research centers.
L'archive ouverte pluridisciplinaire HAL, est destinée au dépôt et à la diffusion de documents scientifiques de niveau recherche, publiés ou non, émanant des établissements d'enseignement et de recherche français ou étrangers, des laboratoires publics ou privés. 


\title{
MEDICAL KNOWLEDGE MODELING IN A SYMBOLIC-CONNECTIONIST PERSPECTIVE
}

\author{
V. Rialle ${ }^{1}$, M. Ohayon ${ }^{2}$, B. Amy ${ }^{3}$, P. Bessière ${ }^{3}$ \\ 1: Faculté de Médecine de Grenoble, Université J. Fourier, Lab. TIMB-IMAG, 38700 La Tronche, France. \\ 2: Centre de Recherche, Hôp. L.H. Lafontaine, 7401 rue Hochelaga, Montréal, P.Q., H1N-3M5, Canada. \\ 3: Université J. Fourier,Laboratoires LIFIA-IMAG et LGI-IMAG,BP 53 X,38041 Grenoble Cedex, France.
}

\begin{abstract}
In this study, we show the specific and complementary attributes of Artificial Intelligence (AI) and of Connectionism (C). AI seems to be more adapted to modeling upper levels of data and knowledge processing performed by the brain, whereas $\mathrm{C}$ is more generally linked to sensory perception, reflexes or pattern recognition processes. A certain number of medical diagnosis aiding systems, combining these two paradigms, document the thesis that hybrid symbolicconnectionist architectures offer a very promising opening for the realization of complex, hight level decision making systems in the years to come.
\end{abstract}

\section{INTRODUCTION}

The methodological difficulties met in building Knowledge Based Systems (KBS) are well-known today. The physician or the cognitive engineer are tempted to resort to Connectionism to improve their methodology in associating Neural Networks (NN) to symbolic Knowledge Bases (KB). The present study aims at underlining the specific features of the connectionist approach versus the symbolic approach of cognitive modeling and, beyond their differences, at showing their complementarity [13] in building Hybrid Symbolic-Connectionist Systems (HSCS) in the field of medicine.

\section{SYMBOLIC PARADIGM}

The specific objectives of Artificial Intelligence [7] can be expressed in a few essential points:

1) give precedence to a declarative form over any other form of knowledge expression. This principle allows both to easily update knowledge (addition, modification, deletion) and to represent it in natural language or graphic form;

2) an AI system requires a considerable amount of knowledge to be declared. Indeed, to the basic domain knowledge must be added numerous secondary knowledge which is often obvious to the expert;

3) explain in plain language all reasonings and inferences (proscription of the black box concept).

\section{CONNECTIONIST PARADIGM}

The connectionist paradigm is based on:

1) mathematical modeling of the neuron by a processing unit usually having several inputs, one output and an activation function [8] which computes the output signal according to the inputs;

2) the connection of such processing units in networks and the study of emergent collective properties of these networks. Such networks are self-adaptable, modifyable by training, and are naturally massively parallel. In a comparison which is more an image than a reflection of reality, 
these networks are similar to networks of biological neurons: inputs simulate synapses, the output

simulates the axon and dendrites, and the processing unit simulates the soma.

Connectionism is essentially made up of mathematical models which can be broken down into two main families according to the type of training (supervised or non-supervised) and into a large number of network models such as mono or multi-layered NN, partially or entirely connected NN, etc.

\section{DIFFERENCES}

$\mathrm{AI}$ and $\mathrm{C}$ correspond to two fundamentally different paradigms [9]:

a) AI postulates a knowledge representation by means of symbols i.e. elements of a language, and therefore makes a basic a priori statement: knowledge can be parted into discrete fragments and represented in the form of symbolic units which are then to be automatically interpreted;

b) the connectionist paradigm is based on the neuron metaphor, imitating to a certain extent the functions of biological neurons.

In cognitive modeling, $\mathrm{AI}$ is generally associated with upper levels of data processing performed by the brain, whereas $\mathrm{C}$ is more generally linked to perception processes, reflexes, pattern recognition, with no intervention of complex reasoning. A NN possesses a knowledge, but this knowledge is not easy to represent since it is distributed along the synaptic weights and the activation functions, and it depends on the architecture or connectivity pattern of the network, on the number of cells, etc.

\section{a. Learning and flexibility}

A certain disenchantment towards AI stems from the non-flexible aspect of concepts locked into symbols: symbolic knowledge representation is rigid by nature and is poorly adapted to noticeable modifications of the model. When a production rule is added or withdrawn, the effects on the whole reasoning process or on the overall coherence of the $\mathrm{KB}$ can be considerable and difficult to master. This rigid feature makes the design of automatic knowledge learning models very difficult. On the contrary, learning is an integral part of bringing $\mathrm{NN}$ into play. However, this comparison has to be greatly attenuated by the difference of conceptual levels specific to both approaches: a learning process of complex reasonings has very little relationship with a learning process of conditioned reflexes.

\section{b. Addressing versus association}

In AI, memory is designed as an addressable topographical structure: an information is memorized in a specific location of that structure, the only means of accessing it being its address. In the case of $\mathrm{C}$, the concept of location of a memorized information disappears and makes place for the concept of associative access to this information: accessing is performed by giving the network a fragment of information (auto-associative memory) or an associated information (hetero-associative memory). As stated above for knowledge, information is generally delocalized, i.e. distributed along the synaptic weights, the activation functions, the type of connectivity and any other characteristic of the network.

\section{c. Dialogue and explanation capabilities}

Classical AI however presents an indisputable advantage over connectionism in the area of hight level knowledge representation and complex reasoning processes in that it allows, on one hand, explanation of deductive processes in plain language and, on the other hand, it easily provides dialogues with the user in natural language [7]. At least historically, connectionism did not have such goals and cannot be compared to AI in that area.

\section{SYMBOLIC-CONNECTIONIST SYSTEMS}

Connectionism studies more and more the emergence of symbols from cells interconnections [3,11]. Practically, hybrid architectures [5] of KBS have been proposed over the last few years and may be classified around four levels of inter-relation [2]: loose coupling, tight coupling, full integration and transformation.

An example of tight coupled system: MACIE, developed by GALLANT [4] is applied to the diagnosis and treatment of acute sarcophagal disease. In this model, a strong symbiosis exists 
between the logic and connectionist levels: a linear discriminant multi-layered network constitutes the very basis of the KB. This system possesses the fundamental features of an expert system (forward and backward chaining, reasoning processes explanation, computation and propagation of likelihood coefficients, etc..

A loosely coupled system applied to the diagnosis of malevolent disease of mammae is proposed by KASABOV [6]. This system is based on production rules (PR) coupled to connectionist networks. PR's are used to represent high level knowledge whereas NN aims at modeling classification or pattern recognition processes.

TIAN HE and TAI JUWEI [12] used a multi-layered NN and applied the backpropagation learning model to construct a KBS in the area of traditional chinese medicine for children cough disease. The related NN is used both for automatic knowlege acquisition and for deep knowledge modeling. ANTONY et al. [1] studied the use of NN for an automatic reporting ES aimed at scanning medical images, extracting relevant features and giving a diagnostic output in nuclear medicine image analysis.

Some other hybrid expert systems exist (inside and outside the medical field) which cannot be listed here in an exhaustive manner. As far as we are concerned, we are building a loosely coupled system to reinforce knowledge related to threshold values of quantitative electromyographic data for their semantic interpretation in a heterogeneous knowledge based system [10].

\section{CONCLUSION}

We have summarized the characteristics of two fundamentally different approaches of knowledge and reasoning modeling. If these approaches are so wide apart, it is mainly due to the fact that they each address different levels of cognition. One should therefore not be surprised if, although rather in opposition, they are nevertheless complementary. The mastery of cognitive modeling then consists of relating and harmonizing them into coherent meta-systems designed to be more efficient than simple AI or connectionism based systems. We illustrated this viewpoint through a few relevant systems pertaining to the medical field. It goes without saying that all scientific fields manipulating large-scale knowledge bases are concerned by HSCS. This type of system still poses however numerous theoretical and methodological questions. For instance, isn't there a risk of coming up against old daemons of purely symbolic systems, such as: limited fields of knowledge, problems of medical validation and insertion into current practice, etc ? On a theoretical level, one can notably wonder what types of relations can be established between connectionism and first order logic, or further: is it possible to offer a theoretical frame common to both AI and C ? In any case, the confrontation and possible merge of these two approaches should expand each of their limits and open the way to richer cognitive models.

\section{REFERENCES}

1. Antony D., Hines E., Taylor D. and Barham J., An investigation into the use of neural networks for an expert system in nuclear medicine image analysis, Third Int. Conf. Image Processing and its Applications (IEE, London, UK, 1989), 338-342.

2. Bailey D.L. and Fahey J.L., Combining Neural and Symbolic Processing, Tutorial 6, Int. Conf. NEURO NIMES '90 (EC2, Nanterre, France, 1990).

3. Feldman J.A., Connectionist Representation of Concepts, in: R. Pfeifer, Z. Schreter, F. Fogelman-Soulié and L. Steels (Eds), Connectionism in perspective (North-Holland Publishing, Amsterdam, Netherland, 1989), 25-45.

4. Gallant S.I., Connectionist expert systems, Communications of the ACM, 2 (31), 1986, 152-169.

5. Hendler J.A., On the need of hybrid systems, Connection Science (special issue on Hybrid Connectionist / Symbolic Systems), 1 (3), 1989.

6. Kasabov N.K., Hybrid connectionist rule-based systems, in: Ph. Jorrand and S. Segurev (Eds.), Artificial Intelligence IV: Methodology, Systems, Applications (Elsevier Science Pub., North-Holland, 1990), 227-235.

7. Kodratoff Y., Enlarging Symbols to more than numbers or Artificial Intelligence Is the Science Explanation, in: R. Pfeifer, Z. Schreter, F. Fogelman-Soulié and L. Steels (Eds), Connectionism in perspective (North-Holland Publ., Amsterdam, Netherlands, 1989) 157-172. 
8. McCulloch W. and Pitts W., A logical calculus of the ideas immanent in nervous activity, Bulletin of Mathematical Physics, 5, 1943, 115-133.

9. Memmi D., Connectionism and Artificial Intelligence, Proc. NEURO-NIMES '89 (EC2, Nanterre, France, 1989), 17-34.

10. Rialle V., Vila A., Besnard Y., Heterogeneous knowledge representation using a finite automaton and first order logic: a case study in electromyography, Art. Intell. in Med., 3 (2), (Elsevier Science Pub., Amsterdam, NorthHolland, 1991), in press.

11. Rumelhart D. E., McClelland J. L. \& the PDP Research Group, Parallel Distributed Processing: Explorations in the Microstructure of Cognition, Vol. 1: Fondations (MIT Press/Bradford Books, Cambridge, Ma, 1986).

12. Tian He and Tai Juwei, Connectionist traditional Chinese medicine expert system - NCCS, in: B. Barber, D. Cao, Qin, D. and Wagner G. (Eds), Proc. MEDINFO 89 - Sixth Int. Conf. on Medical Informatics (North-Holland, Amsterdam, Netherlands), 1186.

13. Zeidenberg M., Neural Networks in Artificial Intelligence (Ellis Horwood, New York, 1990).

V. Rialle : Tel. (+33) $76637147 \quad$ Fax : (+33) $76518667 \quad$ E.mail: rialle@timb.imag.fr 